\title{
Downregulation of microRNA-1 in esophageal squamous cell carcinoma correlates with an advanced clinical stage and its overexpression inhibits cell migration and invasion
}

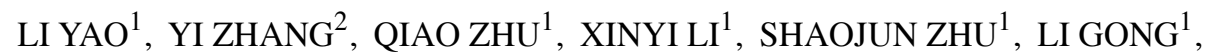 \\ XIUJUAN HAN ${ }^{1}$, MIAO LAN ${ }^{1}$, SHANQU LI $^{3}$, WEI ZHANG ${ }^{1}$ and YANHONG $\mathrm{LI}^{4}$ \\ ${ }^{1}$ Department of Pathology, Tangdu Hospital, The Fourth Military Medical University, Xi'an, Shaanxi 710038; \\ ${ }^{2}$ Cell Engineering Research Center, The Fourth Military Medical University, Xi'an, Shaanxi 710032; \\ ${ }^{3}$ Outpatient Department, and ${ }^{4}$ Department of Gynecology and Obstetrics, Tangdu Hospital, \\ The Fourth Military Medical University, Xi'an, Shaanxi 710038, P.R. China
}

Received June 8, 2014; Accepted January 19, 2015

DOI: 10.3892/ijmm.2015.2094

\begin{abstract}
Esophageal squamous cell carcinoma (ESCC) is one of most common and fatal forms of cancer worldwide. Recent studies have suggested that an aberrant microRNA (miRNA or miR) expression signature exists in ESCC. In the present study, in order to determine the involvement of miRNA in the development and progression of ESCC, the expression profiles of miRNA in 8 paired ESCC tissues and corresponding normal esophageal tissues were analyzed by miRNA microarray. A total of 43 differentially expressed miRNAs, including 27 downregulated and 16 upregulated miRNAs were found in the ESCC tissue samples. Among these miRNAs, we found that miR-1 was significantly downregulated. Subsequently, the expression of miR-1 was validated in 64 pairs of primary ESCC samples by RT-qPCR. The expression level of miR-1 was found to be frequently decreased, and significantly correlated with tumor invasion and an advanced clinical stage $(\mathrm{P}=0.022$ and $\mathrm{P}=0.028$, respectively). In addition, functional assays revealed that miR-1 inhibited cell proliferation, clonogenicity, cell invasion and migration. Bioinformatics analyses identified the major biological processes that were targeted by miR-1. These results suggest that miR-1 has a tumor-suppressive effect on the development and progression of ESCC. The findings of this study may contribute to the further understanding of the functions of miR-1 in ESCC.
\end{abstract}

Correspondence to: Professor Wei Zhang, Department of Pathology, Tangdu Hospital, The Fourth Military Medical University, 569 Xinsi Road, Xi'an, Shaanxi 710038, P.R. China

E-mail: zhangyi@fmmu.edu.cn

Professor Yanhong Li, Department of Gynecology and Obstetrics, Tangdu Hospital, The Fourth Military Medical University, 569 Xinsi Road, Xi'an, Shaanxi 710038, P.R. China

E-mail: liyanhongfmmu@gmail.com

Key words: esophageal squamous cell carcinoma, microRNA-1, migration, invasion, bioinformatics analyses

\section{Introduction}

Esophageal cancer is the sixth most common cause of cancer mortality and the eighth most common type of cancer worldwide $(1,2)$. Esophageal cancer can be divided into 2 main forms: esophageal squamous cell carcinoma (ESCC) and adenocarcinoma (3). Adenocarcinoma is common in Western countries, but ESCC is predominant in East Asia, particularly in China $(2,4)$. Despite advances in the diagnosis and treatment of this type of cancer, the survival rate at 5 years is poor (5). Thus, there is an urgent need for the discovery of novel biomarkers and therapeutic targets for ESCC.

MicroRNAs (miRNAs or miRs) are a class of singlestranded, non-coding RNAs. They are 17-25 ribonucleotides in length $(1,6)$. They function as regulators of gene expression and influence a wide variety of biological processes, including proliferation, differentiation, apoptosis and development (7). miRNAs suppress gene expression at the post-transcriptional level by binding to the 3'-untranslated region (UTR) of target mRNAs with the 'seed sequence' of the RNA-induced signaling complex (RISC) (8). Depending on the degree of complementarity between the miRNA and its target, mRNA expression is blocked either through direct cleavage or translational inhibition (6). Accumulating evidence indicates that miRNAs are aberrantly expressed in various types of cancers, including $\operatorname{ESCC}(1,6)$. They function as a novel class of oncogenes and tumor suppressor genes, depending on the targets they regulate. For example, miR-21 has been shown to be significantly upregulated in ESCC tissues and to regulate cell proliferation and invasion through the suppression of phosphatase and tensin homolog (PTEN) (9,10). However, miR-203 and miR-205 have been reported to be downregulated in $\operatorname{ESCC}(11,12)$. Thus, miRNAs may provide a readily detectable diagnostic and therapeutic marker for ESCC.

In the present study, a microarray strategy was applied to identify differentially expressed miRNAs in ESCC by comparing the miRNA profiles in tumor tissue samples with those of paired non-tumor tissue samples. A total of 43 differentially expressed miRNAs were detected. The expression level 
and tumor-suppressive function of miR-1, which was frequently found to be downregulated in ESCC, was further characterized.

\section{Materials and methods}

ESCC cell lines and clinical samples. The human ESCC cell line, Eca109, was obtained from the Shanghai Institute of Cell Biology, Chinese Academy of Sciences, and was cultured in RPMI-1640 with $10 \%$ fetal bovine serum (FBS). A total of 64 pairs of human ESCC samples were obtained from patients who underwent surgery at the Tangdu Hospital (Xi'an, China) between 2011 and 2012. The corresponding normal esophageal mucosa samples were obtained from the farthest margin of the resected specimen. All the samples were fixed in $10 \%$ formalin and embedded in paraffin. Following H\&E staining, the tumor tissue sections with maximum tumor content were carefully selected. This study was approved by the Research Ethics Review Committee at Tangdu Hospital. All patients signed informed consent approving the use of their tissues for research purposes.

Microarray analysis. Total RNA was extracted and purified from 8 pairs of ESCC tumors and corresponding normal esophageal mucosa samples using a RecoverAll Total Nucleic Acid Isolation kit (AM1975; Ambion, Austin, TX, USA) following the manufacturer's instructions. Prior to microarray analysis, the quality of the RNA was assessed using the Agilent Bioanalyzer 2100 (Agilent Technologies, Santa Clara, CA, USA). The miRNA was distinguished from the remaining RNA using the miRNA Complete Labeling and Hyb kit (Agilent Technologies). Subsequently, each miRNA microarray slide (Agilent Version 16.0) was hybridized with 100 ng labeled RNA and scanned using an Agilent Microarray Scanner (Agilent Technologies). The data were normalized and analyzed using the quantile algorithm and Gene Spring Software 11.0 (Agilent Technologies). Hierarchical cluster analysis was performed using Cluster 3.0 software. The fold change was calculated by comparing the expression level of miRNAs in the ESCC tumor pool to that of the normal tissue pool.

RNA extraction. Total RNA was extracted from the paraffinembedded samples using a miRNeasy FFPE kit (Qiagen, Valencia, CA, USA). A total of 8 sections of $5 \mu \mathrm{m}$ in thickness were freshly cut and placed in a $1.5 \mathrm{ml}$ microcentrifuge tube. Subsequently, $1 \mathrm{ml}$ xylene was added for deparaffinization. The samples were then washed in ethanol washes. Proteins were degraded by proteinase $\mathrm{K}$ digestion and genomic DNA was removed with DNase I step. The concentrated RNA was purified using RNeasy MinElute Spin Columns and eluted in $20 \mu \mathrm{l}$ RNase-free water. The quality and quantity were measured using a NanoDrop spectrophotometer (Thermo Scientific, Waltham, MA, USA).

Reverse transcription reaction-quantitative (real-time) PCR $(R T-q P C R)$. RNA was reverse transcribed using a miScript Reverse-Transcription kit (Qiagen) for first strand complementary DNA synthesis. The reaction conditions involved the incubation of the samples at $37^{\circ} \mathrm{C}$ for $60 \mathrm{~min}$ followed by $95^{\circ} \mathrm{C}$ for $5 \mathrm{~min}$. Quantitative PCR (qPCR) was then performed using primers for miR-1 (miScript Primer Assay: MS00008358) and RNU6B (miScript Primer Assay: MS00033740) and the
miScript SYBR-Green PCR kit (both from Qiagen) with the ABI 7500 Fast Real-time PCR System (Applied Biosystems, Carlsbad, CA, USA). Briefly, the samples were incubated at $95^{\circ} \mathrm{C}$ for $15 \mathrm{~min}$, and qPCR was repeated for 40 cycles with incubation at $94^{\circ} \mathrm{C}$ for $15 \mathrm{sec}$ followed by $55^{\circ} \mathrm{C}$ for $35 \mathrm{sec}$ and then at $70^{\circ} \mathrm{C}$ for $30 \mathrm{sec}$. These reactions were run in triplicate.

Cell proliferation assay. The RNA mimic for miR-1 and the mimic negative control were purchased from GenePharma Co., Ltd. (Shanghai, China). The Eca109 cells were transfected with $100 \mathrm{nM}$ mimic or the mimic negative control using Lipofectamine 2000 in accordance with the manufacturer's instructions (Invitrogen, Carlsbad, CA, USA). The expression level of miR-1 was assayed by RT-qPCR. For foci formation assay, 200 transfected cells were seeded in 60-mm Petri dishes. Surviving colonies (50 cells per colony) were counted using Giemsa staining following 14 days in culture. For MTT assay, 1,000 Eca109 cells were plated and transfected with the mimic or the mimic negative control in a 96-well culture dish. The cells were then incubated for $24,48,72,96$ and $120 \mathrm{~h}$ following transfection. The assay was performed by the addition of MTT solution (Sigma-Aldrich, St. Louis, MO, USA) for $4 \mathrm{~h}$ and DMSO for $15 \mathrm{~min}$. The absorbance (OD) of each well was then measured at a wavelength of $490 \mathrm{~nm}$. The experiment was performed in triplicate.

Cell motility and invasion assay. The Eca109 cells were seeded in a 6-well culture plate and transfected with either the miR-1 mimic or the negative control after the cells reached subconfluence. Wounds were created using a $200 \mu \mathrm{l}$ pipette tip. Wound healing was observed by taking photos under a microscope at 0,24 and $48 \mathrm{~h}$ after scratching. Cell invasion assays were performed using chambers consisting of a Transwell pre-coated Matrigel membrane filter ( $8 \mu \mathrm{m}$ pore size; Millipore Corp., Billerica, MA, USA). The transfected cells were harvested and transferred to the upper chamber filled with serum-free medium. In the lower chamber, $10 \%$ FBS-containing medium was added. After $24 \mathrm{~h}$, the invaded cells were stained and 3 randomly selected fields were counted. Duplicate wells for each condition were examined, and each experiment was repeated 3 times.

Normalization and statistical analysis. The expression level was normalized against endogenous small nuclear RNA gene U6 for the miRNAs examined. The relative quantification of miRNA expression was calculated using the $2^{-\Delta \Delta \mathrm{Ct}}$ method. Statistical analysis was carried out using SPSS 13.0 software for Windows. A paired t-test was used to analyze the differences between the tumor and normal tissue. ANOVA was used to evaluate the association between miRNA expression and the clinicopathological characteristics. Differences were considered statistically significant when the P-value was $<0.05$.

Prediction of miRNA target genes and Gene Ontology (GO) analysis. Given the limitations of any single prediction program, TargetScan, PicTar and miRanda were used to analyze the potential target genes of the deregulated miRNAs. To determine which genes were most likely targeted by the given miRNA, the predicted results from the different databases were integrated. GO analysis was applied to analyze the main function of the differentially expressed genes according 
Table I. Differentiated dysregulation of miRNAs in ESCC.

\begin{tabular}{|c|c|c|}
\hline miRNA name & P-value & Fold change \\
\hline \multicolumn{3}{|l|}{ Downregulated } \\
\hline miR-375 & $2.38 \mathrm{E}-05$ & 175.8322 \\
\hline miR-1 & 7.49E-07 & 147.16624 \\
\hline $\operatorname{miR}-133 b$ & $1.95 \mathrm{E}-04$ & 73.55917 \\
\hline $\operatorname{miR}-30 a^{*}$ & 2.64E-08 & 55.60582 \\
\hline $\operatorname{miR}-145^{*}$ & $9.27 \mathrm{E}-05$ & 55.024837 \\
\hline miR-139-5p & $2.66 \mathrm{E}-09$ & 49.534527 \\
\hline miR-338-3p & 7.54E-08 & 40.948067 \\
\hline $\operatorname{miR}-101$ & $9.85 \mathrm{E}-08$ & 31.244616 \\
\hline $\operatorname{miR}-29 c^{*}$ & $3.15 \mathrm{E}-05$ & 23.400047 \\
\hline miR-95 & $2.22 \mathrm{E}-03$ & 12.73129 \\
\hline miR-30a & $3.55 \mathrm{E}-03$ & 8.1511965 \\
\hline miR-145 & $1.50 \mathrm{E}-05$ & 8.024183 \\
\hline $\operatorname{miR}-4324$ & $1.06 \mathrm{E}-03$ & 7.0982347 \\
\hline $\operatorname{miR}-143$ & 9.69E-06 & 6.7646494 \\
\hline miR-497 & $2.58 \mathrm{E}-04$ & 4.562447 \\
\hline \multicolumn{3}{|l|}{ Upregulated } \\
\hline miR-196a & $3.17 \mathrm{E}-04$ & 69.18334 \\
\hline $\operatorname{miR}-424$ & $6.59 \mathrm{E}-04$ & 30.001791 \\
\hline miR-96 & $6.87 \mathrm{E}-04$ & 26.955471 \\
\hline $\operatorname{miR}-130 b$ & 3.91E-03 & 20.232624 \\
\hline miR-3907 & $2.63 \mathrm{E}-03$ & 12.135655 \\
\hline $\operatorname{miR}-34 b^{*}$ & $9.37 \mathrm{E}-03$ & 9.071809 \\
\hline $\operatorname{miR}-183$ & 2.29E-03 & 8.742586 \\
\hline $\operatorname{miR}-185$ & $1.41 \mathrm{E}-02$ & 7.89331 \\
\hline $\operatorname{miR}-21$ & 7.96E-08 & 4.416791 \\
\hline
\end{tabular}

miRNA, microRNA; ESCC, esophageal squamous cell carcinoma.

to the GO database. DAVID 6.7 was used to annotate the molecular functions of the putative miRNA-targeted genes.

\section{Results}

Differential expression of miRNAs detected in ESCC. To investigate the role of miRNAs in ESCC, the Agilent Human miRNA microarray (version 16.0) containing 1,205 human miRNAs was performed to compare the miRNA profiles between the ESCC tumor samples and the corresponding normal esophageal mucosa samples pooled from 8 primary cases of ESCC. A paired t-test was used to assess the differentially expressed miRNAs between the cancer and normal esophageal tissue samples and differences at $\mathrm{P}<0.05$ were considered statistically significant. With the 2-fold cut-off point, 27 downregulated and 16 upregulated miRNAs were detected in the ESCC tumor tissue samples compared with their normal counterparts (Fig. 1A). Hierarchical cluster analysis of these 43 miRNAs among the 8 pairs of test samples was performed. The analysis revealed that the cancer tissue samples were grouped separately from the normal esophageal tissue samples and this created 2 major cluster branches (Fig. 1B). However, with the 4-fold cut-off point, 15 downregulated and 9 upregulated miRNAs were detected in the ESCC tumor tissue samples compared with their normal counterparts (Table I).
Table II. Association between the expression levels of miR-1 and clinicopathological characteristics.

\begin{tabular}{|c|c|c|}
\hline Characteristics & Cases & $\begin{array}{l}\text { miR-1 } \\
\text { P-value }\end{array}$ \\
\hline \multicolumn{3}{|l|}{ Age (years) } \\
\hline$\leq 63$ & 31 & \multirow[t]{2}{*}{0.333} \\
\hline$>63$ & 33 & \\
\hline \multicolumn{3}{|l|}{ Gender } \\
\hline Male & 49 & \multirow[t]{2}{*}{0.354} \\
\hline Female & 15 & \\
\hline \multicolumn{3}{|l|}{ Tumor invasion } \\
\hline $\mathrm{T} 1$ & 13 & \multirow[t]{3}{*}{$0.022^{\mathrm{a}}$} \\
\hline $\mathrm{T} 2$ & 19 & \\
\hline $\mathrm{T} 3$ & 32 & \\
\hline \multicolumn{3}{|c|}{ Lymph node metastasis } \\
\hline No & 45 & \multirow[t]{2}{*}{0.335} \\
\hline N1-N4 & 19 & \\
\hline \multicolumn{3}{|l|}{ Differentiation } \\
\hline I-II & 27 & \multirow[t]{2}{*}{0.365} \\
\hline II-III & 37 & \\
\hline \multicolumn{3}{|l|}{ Clinical stage } \\
\hline I & 10 & \multirow[t]{3}{*}{$0.028^{\mathrm{a}}$} \\
\hline II & 36 & \\
\hline III & 18 & \\
\hline \multicolumn{3}{|l|}{ Tumor size } \\
\hline$\leq 5 \mathrm{~cm}$ & 36 & \multirow[t]{2}{*}{0.441} \\
\hline$>5 \mathrm{~cm}$ & 28 & \\
\hline
\end{tabular}

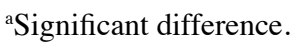

To confirm the results from microarray analysis, 5 downregulated miRNAs (miR-375, miR-1, miR-133b, miR-30a* and miR-101) and 4 upregulated miRNAs (miR-196a, miR-424, miR-96 and miR-130b) were selected for validation by RT-qPCR in the same 8 pairs of test samples. All the miRNAs examined showed a pattern of up- or downregulation similar to that obtained by microarray analysis (Fig. 1C).

Downregulation of miR-1 in ESCC. RT-qPCR was performed to confirm the expression levels of miR-1 in 64 pairs of primary ESCC samples. The fold change was calculated by comparing the relative expression level of miR-1 in the cancer tissue samples and corresponding normal esophageal tissue samples. The small nuclear RNA gene U6 served as a reference gene. The results revealed that miR-1 was significantly downregulated in the primary ESCC samples compared with the corresponding normal esophageal tissue samples. Among all the samples, 90.6\% (58/64) of the ESCC tissue samples showed a 2-fold lower expression of miR-1 (Fig. 2).

In addition, in order to determine the clinical significance of the downregulation of miR-1 in primary ESCC, the correlation of miR-1 downregulation with clinicopathological characteristics from 64 patients was investigated. The results revealed that the downregulation of miR-1 significantly correlated with tumor invasion and an advanced clinical stage $(\mathrm{P}=0.022$ and $\mathrm{P}=0.028$, respectively; Table II), while 

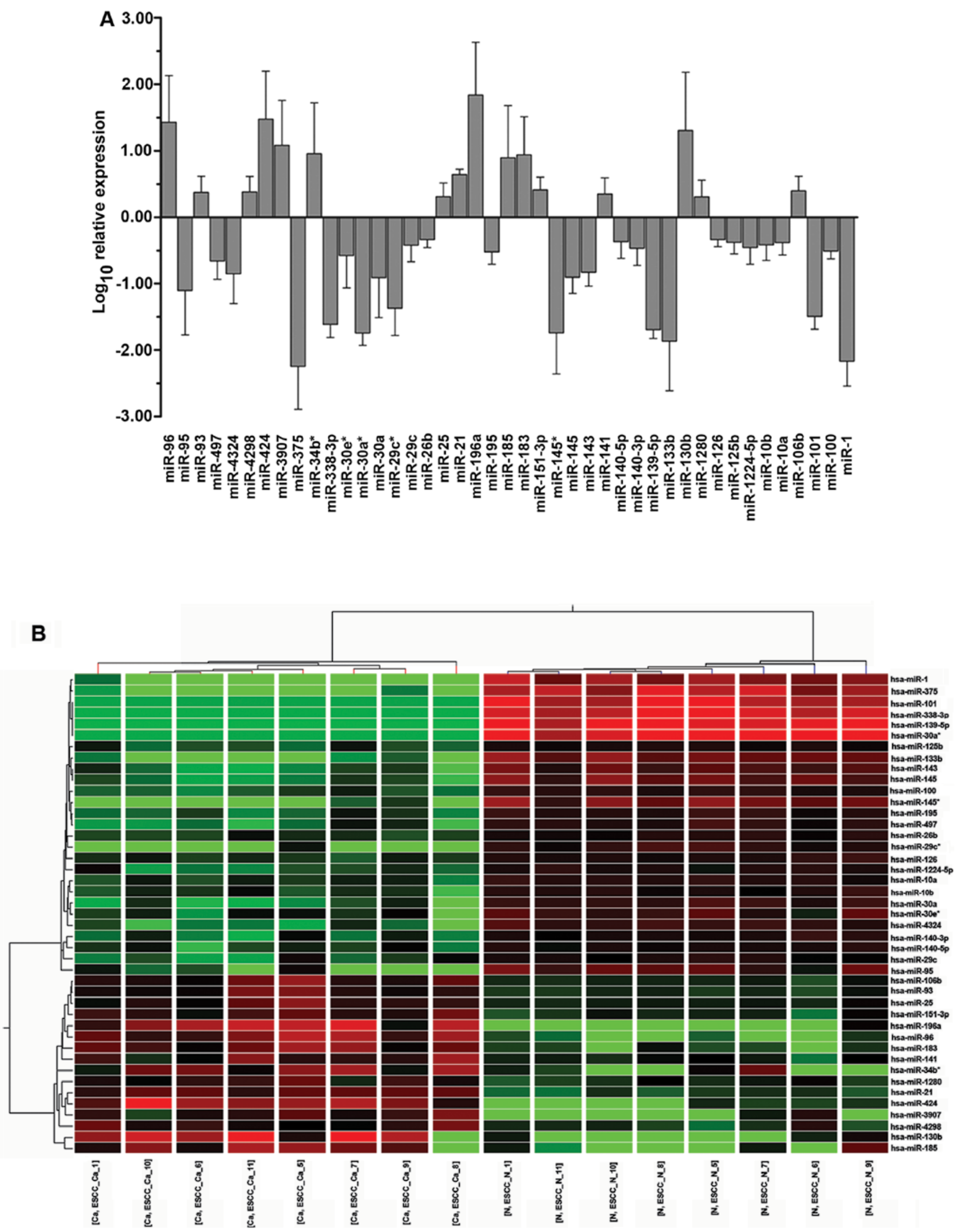

Figure 1. Differentially expressed miRNAs in esophageal squamous cell carcinoma (ESCC). (A) Expression of the miRNAs in ESCC tissue samples compared with the corresponding normal tissue samples by miRNA microarray. (B) Unsupervised hierarchical cluster analysis of miRNA expression. The red and green colors indicate a relatively high and low expression, respectively. The 2 major subgroups distinguished the ESCC samples from cancer samples and corresponding normal esophageal tissue samples. 


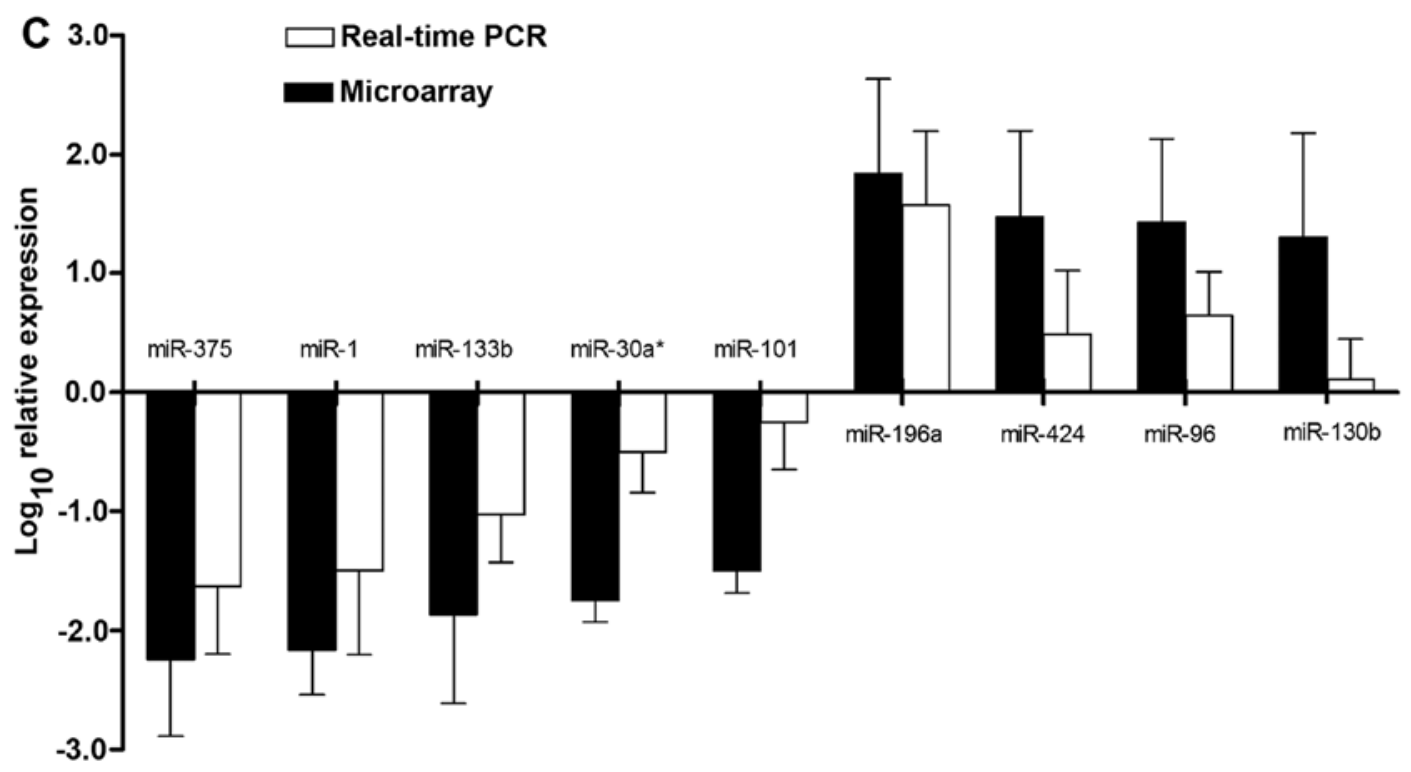

Figure 1. Continued. (C) Confirmation of microarray results by RT-qPCR. A total of 5 downregulated miRNAs (miR-375, miR-1, miR-133b, miR-30a* and miR-101) and 4 upregulated miRNAs (miR-196a, miR-424, miR-96 and miR-130b) were selected and validated in the ESCC samples used for microarray. Fold change of miRNAs was calculated using the $2^{-\Delta \Delta \mathrm{Ct}}$ method and is presented in the $\log _{10}$ scale.

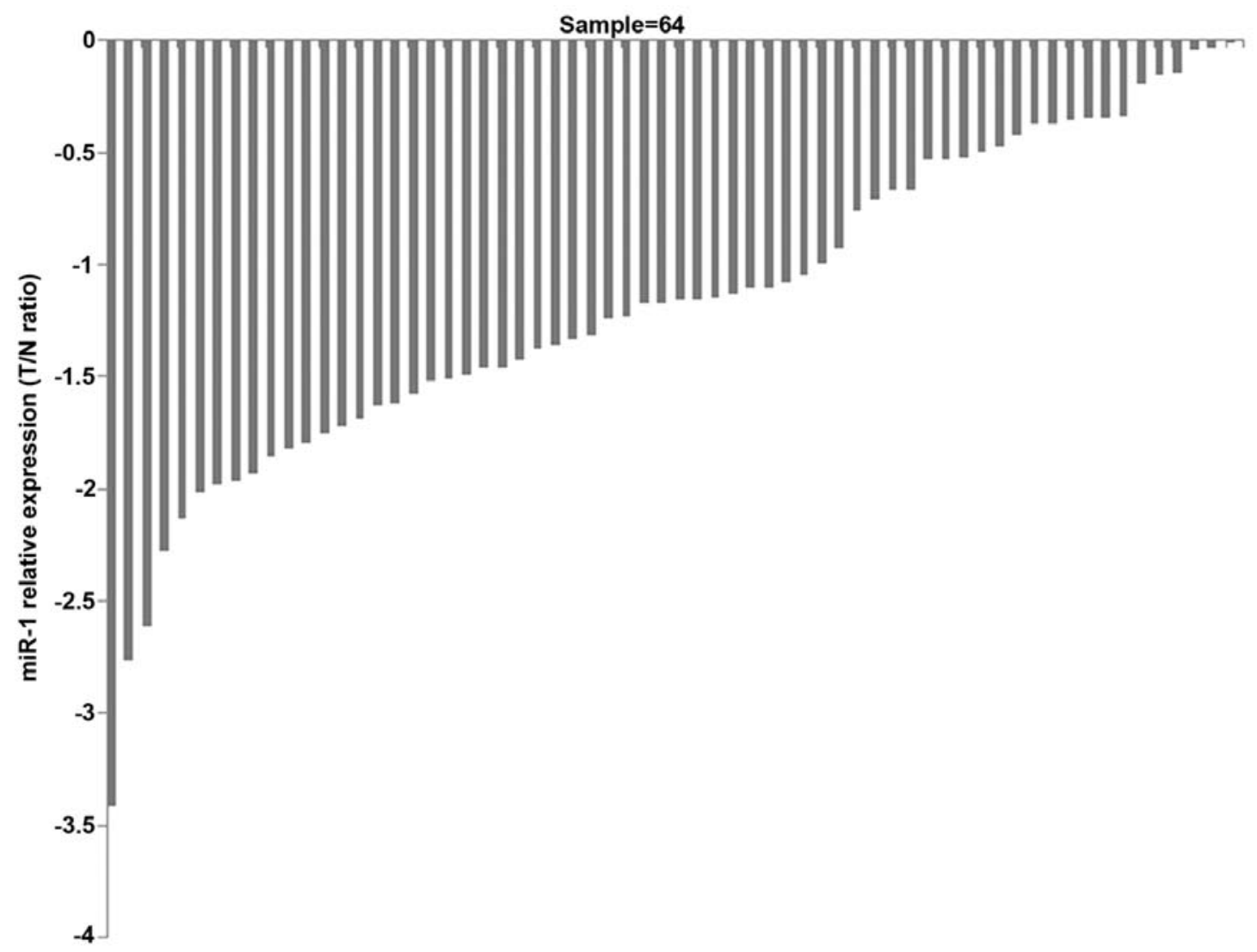

Figure 2. miR-1 was significantly downregulated in the 64 primary esophageal squamous cell carcinoma (ESCC) samples compared with their corresponding normal tissue samples. Using the $2^{-\Delta \Delta C t}$ method, those with a $>2$-fold change were considered significant. The expression of miR-1 was downregulated in $90.6 \%$ $(58 / 64)$ of the ESCC tissue samples. Expression is shown in the $\log _{10}$ scale and was normalized against the endogenous control small nuclear RNA gene U6.

no significant correlation was observed with respect to other clinicopathological characteristics. These results suggest that the downregulation of miR-1 plays an important role in the development of ESCC. 

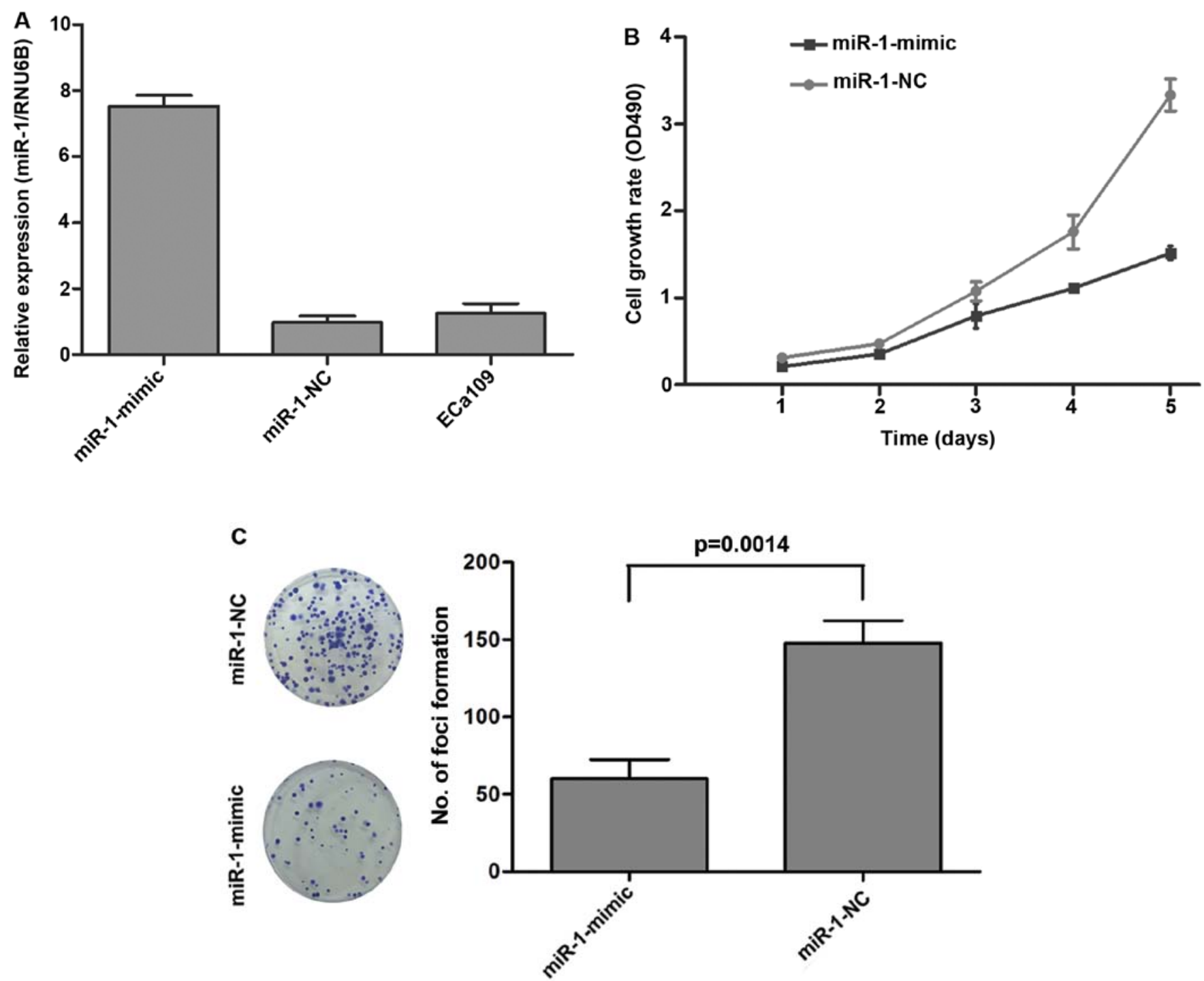

Figure 3. Tumor suppressive functions of miR-1. (A) The efficiency of miR-1 mimics was validated by RT-qPCR. (B) Cell growth rates were suppressed by miR-1 as detected by MTT assay. (C) miR-1 inhibited the foci formation ability of Eca109 cells. All the results are expressed as the means \pm SD.

miR-1 suppresses tumor cell growth. To explore the potential tumor-suppressive role of miR-1 in ESCC, the synthetic miR-1 mimic was transfected into the ESCC Eca109 cells. The expression of mature miR-1 was validated by RT-qPCR (Fig. 3A). MTT assay was used to evaluate cell viability. The assay revealed that the ectopic expression of miR-1 inhibited the cell growth rate (Fig. 3B). To further determine the effects of miR-1 on the growth of ESCC cells, a colony formation assay was performed. The results revealed that the colony-forming ability of the ESCC cells transfected with miR-1 declined by approximately $40 \%$ compared with the cells transfected with the negative control (Fig. 3C).

miR-1 modulates cell migration and invasion. In addition to cell growth inhibition, the effects of miR-1 on tumor migration and invasion were also investigated in this study. Wound healing assays revealed that the cells transfected with the miR-1 mimic migrated very small distances and were unable to achieve wound closure within $48 \mathrm{~h}$ (Fig. 4A). To validate that the suppression of mobility was not caused by the inhibitory effect on tumor cell growth, a Matrigel invasion assay was also performed. The results revealed that the number of invading cells was significantly decreased in the miR-1-transfected cells (Fig. 4B).

miRNA-targeted gene prediction and GO analysis. In order to investigate the potential target and possible mechanisms underlying the regulatory effects of miR-1 in ESCC, we searched for potential targets using TargetScan, PicTar and miRanda. A total of 584, 535 and 3,017 target genes of miR-1 were predicted using the 3 databases stated above, respectively. Among these, 181 genes were common to all 3 databases. The predicted target genes of miR-1 were then classified by GO enrichment analysis. We observed that the significantly enriched target genes of miR-1 were associated with the following: i) transcription regulator activity; ii) sequence-specific DNA binding; iii) the endomembrane system; iv) regulation of transcription; v) vasculature development (Fig. 5). 

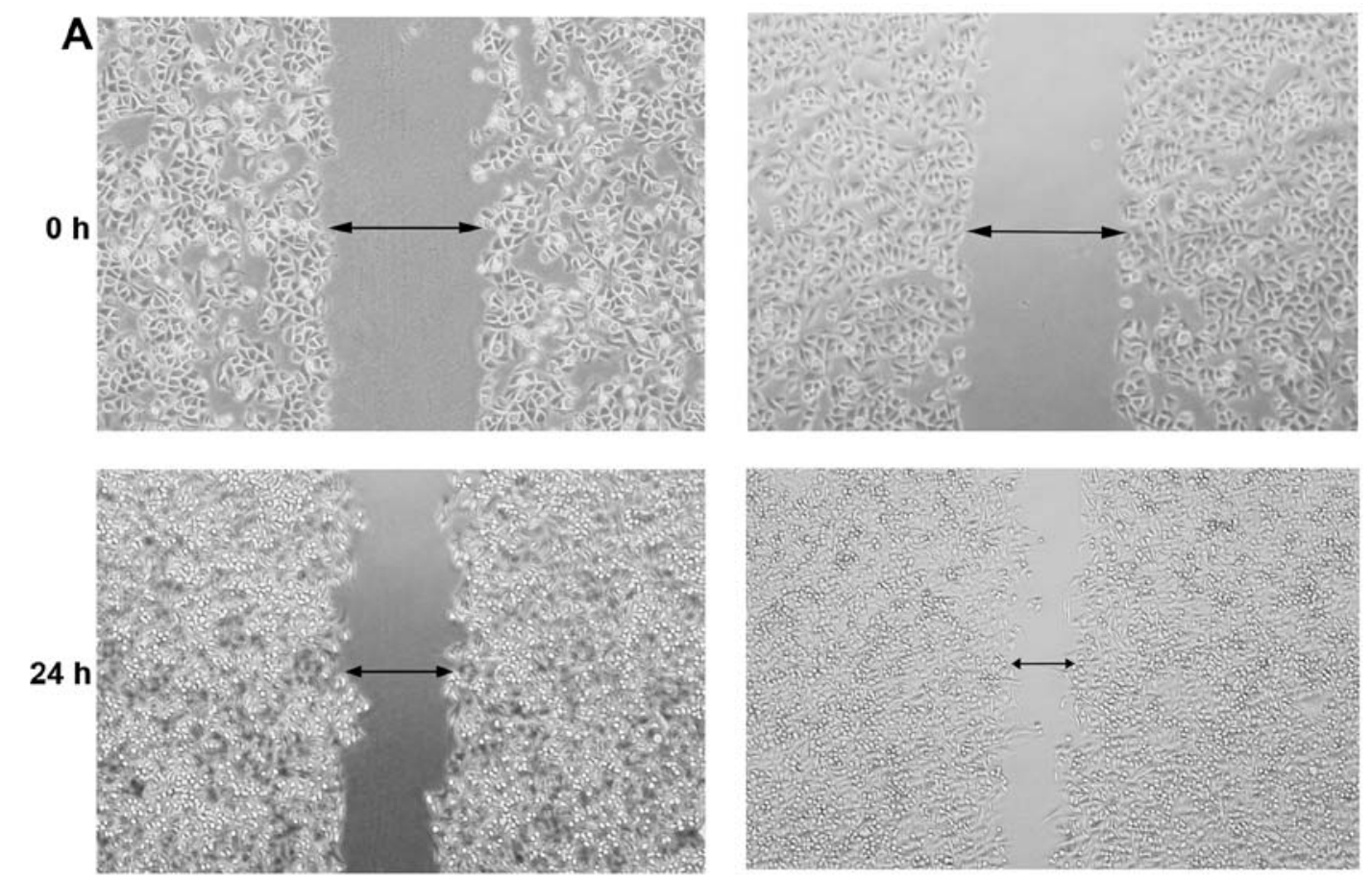

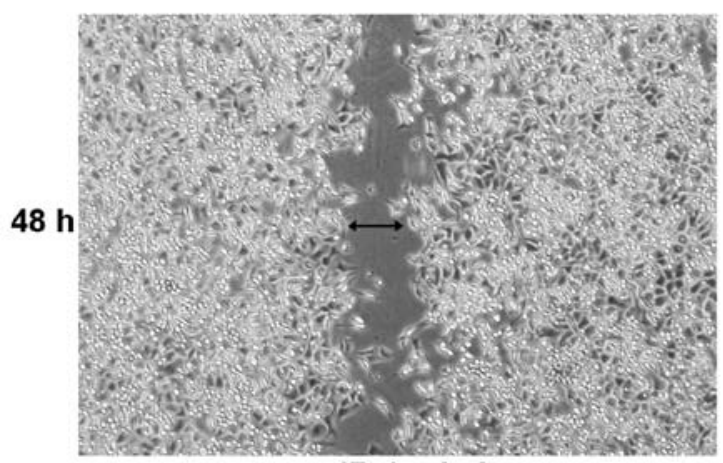

miR-1-mimic

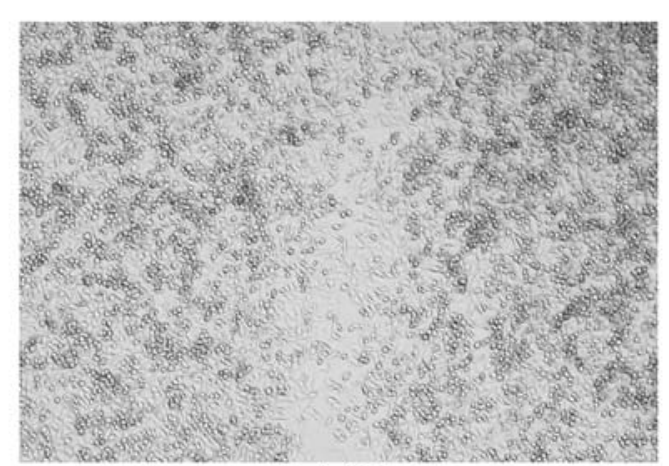

miR-1-NC

B
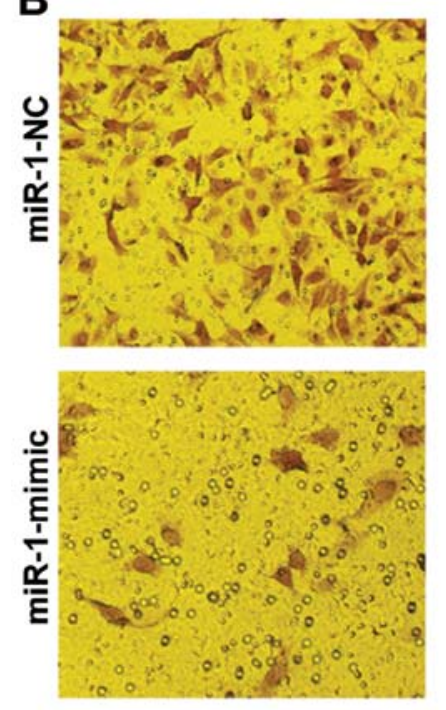

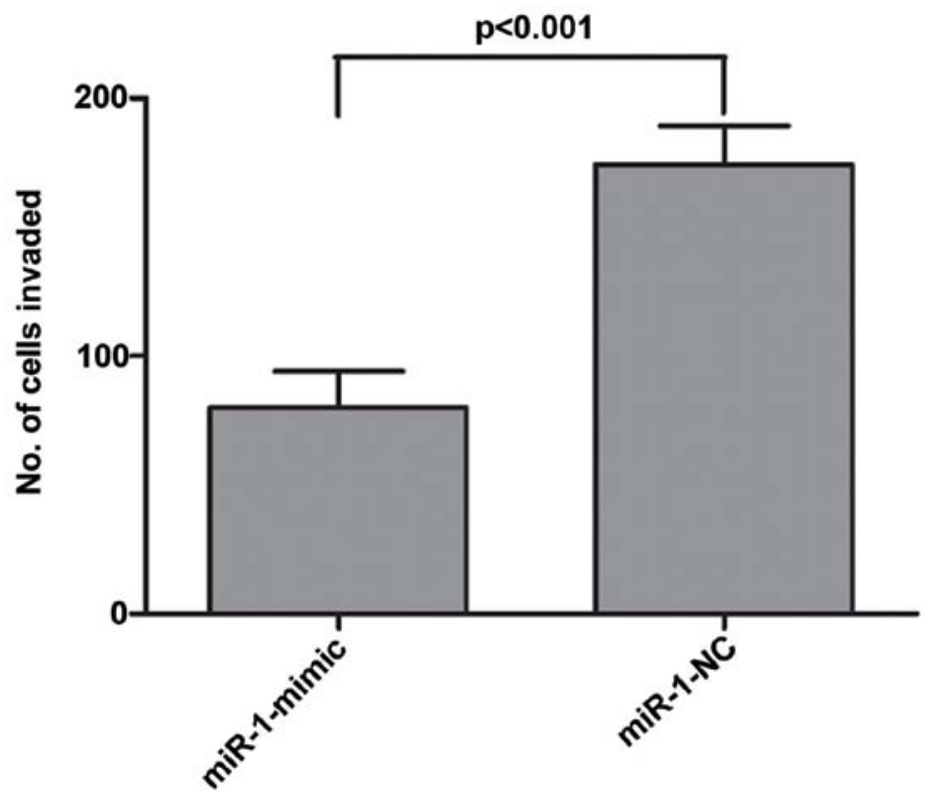

Figure 4. miR-1 modulates cell migration and invasion. (A) Wound healing assays revealed that cell motibily was suppressed by miR-1. Representative images were taken at 0,24 and $48 \mathrm{~h}$ after scratching. (B) Cell invasion assays were performed using Matrigel invasion assay. miR-1 impeded tumor cell invasion in Eca109 cells. All the results are expressed as the means \pm SD. 


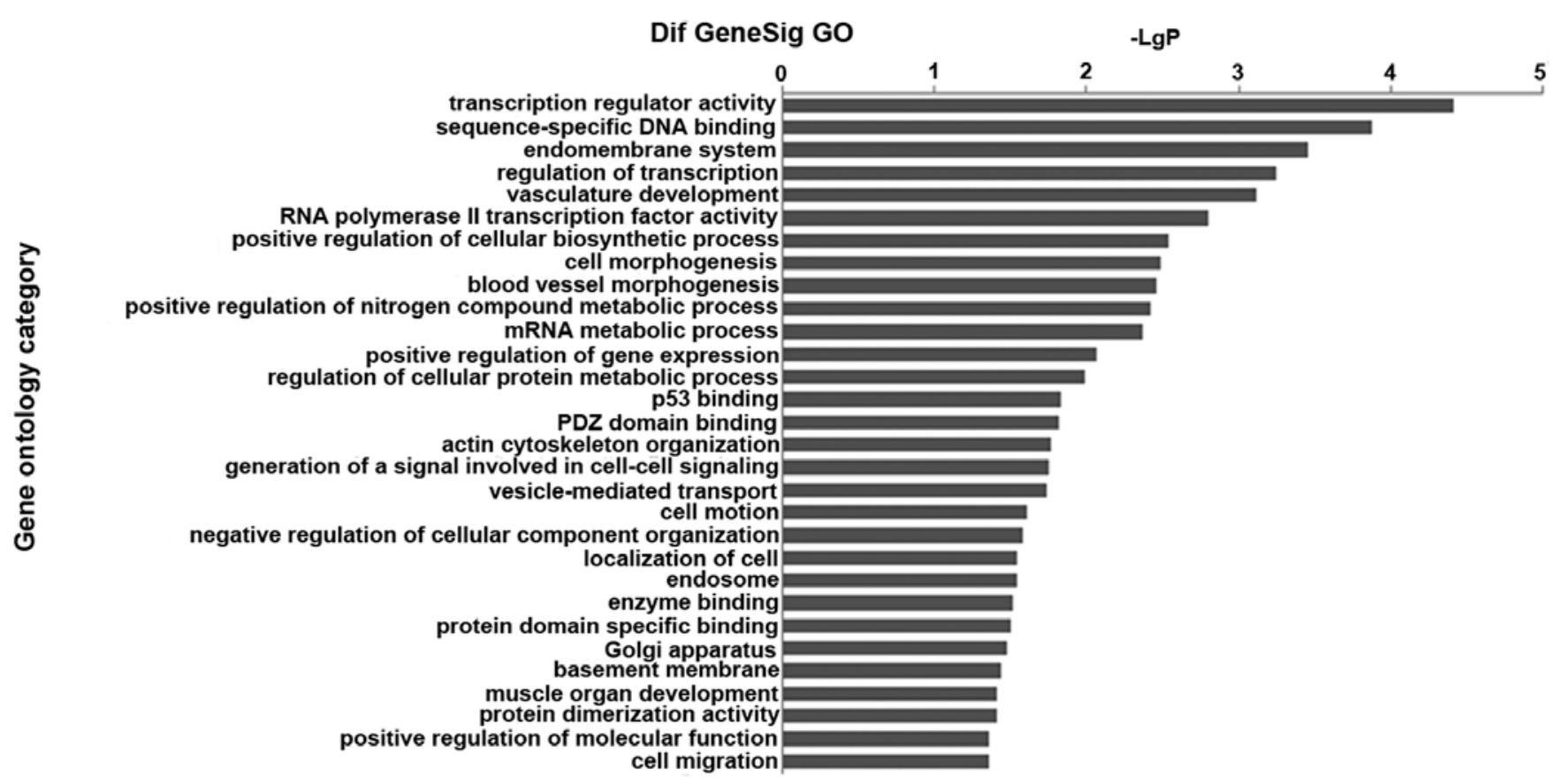

Figure 5. Significant miRNA targets identified by Gene Ontology (GO) analysis. Chart shows the GOs targeted by miR-1. All these GOs showed increased enrichment. The vertical axis is the GO category, and the horizontal axis is the enrichment of GO.

\section{Discussion}

miRNAs have been reported to play critical roles in cancer development and progression through the suppression of downstream target genes (8). The importance of miRNAs in cancer was first recognized when certain miRNAs were found to be specifically deleted in B-cell chronic lymphocytic leukemia (13). Since then, abnormal miRNA expression has been detected in different types of cancer $(3,14)$. However, certain studies have focused instead on the association between miRNA and ESCC (8). In this study, for a better understanding of the molecular mechanisms involved in the development and progression of ESCC, the miRNA profiles in 8 primary ESCC cases were compared. Taking into account the baseline differences between individuals when testing for miRNA differential expression, the tumor and adjacent normal tissues samples were paired. With a 2-fold cut-off point, 43 differentially expressed miRNAs were detected. Following this, we validated 5 downregulated miRNAs (miR-375, miR-1, miR-133b, miR-30a* and miR-101) and 4 upregulated miRNAs (miR-196a, miR-424, miR-96 and miR-130b) in the same 8 pairs of test samples by RT-qPCR. Consistent with the microarray results, all these miRNAs had a similar pattern of up- or downregulation. Due to the absolute fold change, miR-1 was selected for further analysis. miR-1 was originally identified as a muscle-specific miRNA enriched in cardiac and skeletal muscles $(15,16)$. It plays important roles in cardiogenesis and myogenesis (17). More recently, miR-1 has been reported to be downregulated in various types of cancer and to have tumor-suppressive functions (18). For example, miR-1 has been shown to be downregulated and to suppress tumor cell migration through the miR-1-NOTCH3-Asef pathway in colorectal tumor cells (19). It also suppresses transgelin 2 (TAGLN2) in bladder cancer and head and neck squamous cell carcinoma (SCC) $(20,21)$. miR-1 has been shown to suppress the migration and invasion of laryngeal SCC (22). miR-1 also induces apoptosis by targeting prothymosin alpha (PTMA) in a nasopharyngeal carcinoma cell line (23). However, the role of miR-1 in ESCC remains to be elucidated.

In the present study, we found that miR-1 was frequently downregulated in ESCC $(n=64)$ and that the downregulation of miR-1 significantly correlated with tumor invasion and an advanced clinical stage in ESCC. The effects of miR-1 on ESCC development and progression were also investigated by assays. By transfecting miR-1 into an ESCC cell line (Eca109), the expression of miR-1 markedly inhibited the tumor cell growth rate and foci formation. Furthermore, the expression of miR-1 suppressed tumor cell invasion and migration. It is plausible that miR-1 may play an important role as a tumor suppressor in the tumorigenesis and progression of ESCC.

Each miRNA can potentially downregulate several target genes by binding to their 3'-UTR. miRNAs may play different roles in different types of cancer. To identify the downstream target genes regulated by dysregulated miRNAs is an important task. In this study, using the DAVID bioinformatics tool, GO enrichment analysis based on the predicted genes of miR-1 revealed that the mRNA clusters were significantly enriched for transcription regulation and cellular protein metabolic process regulation, protein dimerization activity and the regulation of molecular function, which outline the effects of miR-1 on ESCC.

In conclusion, we demonstrated miRNA expression profiles in ESCC and that miR-1 was markedly downregulated in ESCC, which significantly correlated with tumor invasion and an advanced clinical stage. Furthermore, the enhanced expression of miR-1 inhibited ESCC cell proliferation, invasion and migration, suggesting that miR-1 functions as a tumor suppressor in ESCC. Bioinformatics analyses revealed the 
major biological processes that are targeted by miR-1. Our data present new evidence of the important role of miR-1 in ESCC. Further studies are warranted to perform a more detailed mechanistic analysis of miR-1 and to evaluate the usefulness of miR-1 as a diagnostic/prognostic marker and potential therapeutic target molecules in ESCC.

\section{Acknowledgements}

This study was supported by grants from the National Natural Science Foundation of China (nos. 30800417, 81001088 and 81372226), the National Key Basic Research Program (973 Program) of China (no. 2015CB553703) and the Natural Science Foundation of Shaanxi (no. 2012K13-01-15).

\section{References}

1. Mathé EA, Nguyen GH, Bowman ED, et al: MicroRNA expression in squamous cell carcinoma and adenocarcinoma of the esophagus: associations with survival. Clin Cancer Res 15 6192-6200, 2009.

2. Matsushima K, Isomoto H, Kohno S and Nakao K: MicroRNAs and esophageal squamous cell carcinoma. Digestion 82: 138-144, 2010.

3. Feber A, Xi L, Pennathur A, et al: MicroRNA prognostic signature for nodal metastases and survival in esophageal adenocarcinoma. Ann Thorac Surg 91: 1523-1530, 2011.

4. Su M, Liu M, Tian DP, et al: Temporal trends of esophageal cancer during 1995-2004 in Nanao Island, an extremely high-risk area in China. Eur J Epidemiol 22: 43-48, 2007.

5. Enzinger PC and Mayer RJ: Esophageal cancer. N Engl J Med 349: 2241-2252, 2003.

6. Iorio MV and Croce CM: MicroRNAs in cancer: small molecules with a huge impact. J Clin Oncol 27: 5848-5856, 2009.

7. Ng EK, Chong WW, Jin H, et al: Differential expression of microRNAs in plasma of patients with colorectal cancer: a potential marker for colorectal cancer screening. Gut 58 : $1375-1381,2009$

8. Hui A, How C, Ito E and Liu FF: Micro-RNAs as diagnostic or prognostic markers in human epithelial malignancies. BMC Cancer 11: 500, 2011.

9. Mori Y, Ishiguro H, Kuwabara Y, et al: MicroRNA-21 induces cell proliferation and invasion in esophageal squamous cell carcinoma. Mol Med Rep 2: 235-239, 2009.

10. Ma WJ, Lv GD, Tuersun A, et al: Role of microRNA-21 and effect on PTEN in Kazakh's esophageal squamous cell carcinoma. Mol Biol Rep 38: 3253-3260, 2011.
11. Melar-New M and Laimins LA: Human papillomaviruses modulate expression of microRNA 203 upon epithelial differentiation to control levels of p63 proteins. J Virol 84: 5212-5221, 2010.

12. Matsushima $\mathrm{K}$, Isomoto $\mathrm{H}$, Yamaguchi N, et al: miRNA-205 modulates cellular invasion and migration via regulating zinc finger E-box binding homeobox 2 expression in esophageal squamous cell carcinoma cells. J Transl Med 9: 30, 2011.

13. Calin GA, Dumitru CD, Shimizu M, et al: Frequent deletions and down-regulation of micro- RNA genes $m i R 15$ and $m i R 16$ at 13 q14 in chronic lymphocytic leukemia. Proc Natl Acad Sci USA 99: 15524-15529, 2002.

14. Hamfjord J, Stangeland AM, Hughes T, et al: Differential expression of miRNAs in colorectal cancer: comparison of paired tumor tissue and adjacent normal mucosa using high-throughput sequencing. PLoS One 7: e34150, 2012.

15. Heidersbach A, Saxby C, Carver-Moore K, et al: microRNA-1 regulates sarcomere formation and suppresses smooth muscle gene expression in the mammalian heart. Elife 2: e01323, 2013.

16. Wystub K, Besser J, Bachmann A, Boettger T and Braun T miR-1/133a clusters cooperatively specify the cardiomyogenic lineage by adjustment of myocardin levels during embryonic heart development. PLoS Genet 9: e1003793, 2013.

17. Townley-Tilson WH, Callis TE and Wang D: MicroRNAs 1, 133, and 206: critical factors of skeletal and cardiac muscle development, function, and disease. Int J Biochem Cell Biol 42: 1252-1255, 2010.

18. Tahara H, Kay MA, Yasui W and Tahara E: MicroRNAs in Cancer: the 22nd Hiroshima Cancer Seminar/the 4th Japanese Association for RNA Interference Joint International Symposium, 30 August 2012, Grand Prince Hotel Hiroshima. Jpn J Clin Oncol 43: 579-582, 2013.

19. Furukawa S, Kawasaki Y, Miyamoto M, Hiyoshi M, Kitayama J and Akiyama T: The miR-1-NOTCH3-Asef pathway is important for colorectal tumor cell migration. PLoS One 8: e80609, 2013.

20. Yoshino H,Chiyomaru T,EnokidaH,etal: The tumour-suppressive function of miR-1 and miR-133a targeting TAGLN2 in bladder cancer. Br J Cancer 104: 808-818, 2011.

21. Nohata N, Sone Y, Hanazawa T, et al: miR-1 as a tumor suppressive microRNA targeting TAGLN2 in head and neck squamous cell carcinoma. Oncotarget 2: 29-42, 2011.

22. Wang F, Song G, Liu M, Li X and Tang H: miRNA-1 targets fibronectin1 and suppresses the migration and invasion of the HEp2 laryngeal squamous carcinoma cell line. FEBS Lett 585: 3263-3269, 2011.

23. Wu CD, Kuo YS, Wu HC and Lin CT: MicroRNA-1 induces apoptosis by targeting prothymosin alpha in nasopharyngeal carcinoma cells. J Biomed Sci 18: 80, 2011. 